\title{
Quantum treatment of neutrino in background matter
}

\author{
A I Studenikin \\ Department of Theoretical Physics, Moscow State University, 119992 Moscow, Russia \\ E-mail: studenik@srd.sinp.msu.ru
}

\begin{abstract}
Motivated by the need of elaboration of the quantum theory of the spin light of neutrino in matter $(S L \nu)$, we have studied in more detail the exact solutions of the Dirac equation for neutrinos moving in the background matter. These exact neutrino wave functions form a basis for a rather powerful method of investigation of different neutrino processes in matter, which is similar to the Furry representation of quantum electrodynamics in external fields. Within this method we also derive the corresponding Dirac equation for an electron moving in matter and consider the electromagnetic radiation ("spin light of electron in matter", $S L e$ ) that can be emitted by the electron in this case.
\end{abstract}




\section{Introduction}

The presence of matter has a considerable impact on neutrinos. The resonant neutrino flavour oscillations in matter [1, 2] has been proven to be the mechanism for solving the solar neutrino problem. The resonant neutrino spin (or spin-flavour) oscillations in matter [3, 4] have also important consequences in astrophysics and cosmology (see, for instance, [5]). In the presence of matter a neutrino dispersion relation is modified [6]-8], in particular, it has a minimum at nonzero momentum [9, 10]. As it was shown in [6]-[8], the standard result for the MSW effect can be derived using a modified Dirac equation for the neutrino wave function with a matter potential proportional to the density being added. The problem of a neutrino mass generation in different media was also studied [11, 12] on the basis of modified Dirac equations for neutrinos. On the same basis spontaneous neutrino-pair creation in matter was also studied [13]-[14] (neutrino-pair creation in a perturbative way has been recently discussed in [15]).

In [16, 17] another important effect of the matter influence on neutrinos was considered. It was shown that a majorana neutrino moving in matter can decay into antineutrino with emission of the light scalar majoron: $\nu \rightarrow \tilde{\nu}+\phi$. This process could exist due to the modification of the neutrino spectrum induced by the presence of the background matter. In the case of a high enough density this reaction is open even for very light or massless neutrinos. The total decay width of the process can be written

as $\Gamma=\frac{h^{2}}{8 \pi} V, \quad V=\sqrt{2} G_{F} \frac{\rho_{e f f}}{m_{n}}$ [16, 17], where $h$ is the coupling constant of neutrinos to majorons, $\rho_{\text {eff }}$ is the effective matter density and $m_{n}$ is the neutron mass. It should be noted that the total width does not depend on the neutrino energy, meanwhile it is determined by the density of the background matter. The presence of matter can also induce the decay process of the light majoron into the couple of neutrinos (or antineutrinos) [17]: $\phi \rightarrow 2 \nu$ (or $\phi \rightarrow 2 \tilde{\nu})$.

In a series of our papers [18] the quasi-classical treatment of the background matter influence on neutrinos was developed. In particular, we proposed the generalized Bargmann-Michel-Telegdi equation which we used for description of the neutrino spin evolution in matter. Within this approach we predicted [19] a new type of electromagnetic radiation that can be produced by the Dirac neutrino with nonzero magnetic moment 20] in the background matter (for a recent discussion on the neutrino electromagnetic properties, see [21]). We have termed this radiation the "spin light of neutrino" $(S L \nu)$ in matter. The quasi-classical theory of $S L \nu$ has been developed in [19, 22. However, $S L \nu$ is a quantum phenomenon by its nature, and as it has become clear the quantum treatment of this process should be elaborated [23]-[26].

In this paper we present in more detail the quantum treatment of neutrinos in the presence of a dense background matter which implies the use of neutrino energy spectra and exact solutions of modified Dirac equation for neutrino in matter. The approach developed is similar to the Furry representation of quantum electrodynamics, widely used for description of particle interactions in the presence of external electromagnetic fields. This approach establishes a basis for an effective method for investigations of 
different phenomena which can arise when neutrinos move in dense astrophysical or cosmological media. We show below how the developed quantum description of the neutrino in the presence of the background matter can be used for elaboration of the quantum theory of the spin light of neutrino. Within this method applied to an electron, we also derive the corresponding Dirac equation for an electron moving in matter and consider the electromagnetic radiation (which we termed the "spin light of electron in matter", $S L e)$ that can be emitted in this case.

\section{Neutrino quantum states in matter}

In [23] (see also [24]-26]) we derived the modified Dirac equation for the neutrino wave function exactly accounting for the neutrino interaction with matter. Let us consider the case of matter composed of electrons, neutrons, and protons and also suppose that the neutrino interaction with background particles is given by the standard model supplied with the singlet right-handed neutrino. The corresponding addition to the effective interaction Lagrangian is given by

$$
\Delta L_{e f f}=-f^{\mu}\left(\bar{\nu} \gamma_{\mu} \frac{1+\gamma^{5}}{2} \nu\right), \quad f^{\mu}=\sqrt{2} G_{F} \sum_{f=e, p, n} j_{f}^{\mu} q_{f}^{(1)}+\lambda_{f}^{\mu} q_{f}^{(2)}
$$

where

$q_{f}^{(1)}=\left(I_{3 L}^{(f)}-2 Q^{(f)} \sin ^{2} \theta_{W}+\delta_{e f}\right), q_{f}^{(2)}=-\left(I_{3 L}^{(f)}+\delta_{e f}\right), \delta_{e f}= \begin{cases}1 & \text { for } f=e, \\ 0 & \text { for } f=n, p .\end{cases}$

Here $I_{3 L}^{(f)}$ and $Q^{(f)}$ are, respectively, the values of the isospin third components and electric charges of the particles of matter $(f=e, n, p)$. The corresponding currents $j_{f}^{\mu}$ and polarization vectors $\lambda_{f}^{\mu}$ are

$$
j_{f}^{\mu}=\left(n_{f}, n_{f} \mathbf{v}_{f}\right), \quad \lambda_{f}^{\mu}=\left(n_{f}\left(\boldsymbol{\zeta}_{f} \mathbf{v}_{f}\right), n_{f} \boldsymbol{\zeta}_{f} \sqrt{1-v_{f}^{2}}+\frac{n_{f} \mathbf{v}_{f}\left(\boldsymbol{\zeta}_{f} \mathbf{v}_{f}\right)}{1+\sqrt{1-v_{f}^{2}}}\right),
$$

$\theta_{W}$ is the Weinberg angle. In the above formulas (3), $n_{f}, \mathbf{v}_{f}$ and $\boldsymbol{\zeta}_{f}\left(0 \leq\left|\boldsymbol{\zeta}_{f}\right|^{2} \leq 1\right)$ stand, respectively, for the invariant number densities, average speeds and polarization vectors of the matter components. A detailed discussion on the meaning of these characteristics can be found in [18. Using the standard model Lagrangian with the extra term (11), we derive the modified Dirac equation for the neutrino wave function in matter:

$$
\left\{i \gamma_{\mu} \partial^{\mu}-\frac{1}{2} \gamma_{\mu}\left(1+\gamma_{5}\right) f^{\mu}-m\right\} \Psi(x)=0 .
$$

This is the most general form of the equation for the neutrino wave function in which the effective potential $V_{\mu}=\frac{1}{2}\left(1+\gamma_{5}\right) f_{\mu}$ includes both the neutral and charged current interactions of the neutrino with the background particles and which could also account for effects of matter motion and polarization. It should be mentioned that other modifications of the Dirac equation were previously used in [6]-12] for studies of the neutrino dispersion relations, neutrino mass generation and neutrino oscillations in the 
presence of matter. Note that the corresponding quantum wave equation for a Majorana neutrino can be obtained from (44) via the substitution $1+\gamma_{5} \rightarrow 2 \gamma_{5}$ (see also [27, 28]).

For several important cases the equation (44) can be solved exactly. In particular, in the case of the neutrino motion in matter at rest we get [23]

$$
\Psi_{\varepsilon, \mathbf{p}, s}(\mathbf{r}, t)=\frac{e^{-i\left(E_{\varepsilon} t-\mathbf{p r}\right)}}{2 L^{\frac{3}{2}}}\left(\begin{array}{c}
\sqrt{1+\frac{m}{E_{\varepsilon}-\alpha m}} \sqrt{1+s \frac{p_{3}}{p}} \\
s \sqrt{1+\frac{m}{E_{\varepsilon}-\alpha m}} \sqrt{1-s \frac{p_{3}}{p}} e^{i \delta} \\
s \varepsilon \sqrt{1-\frac{m}{E_{\varepsilon}-\alpha m}} \sqrt{1+s \frac{p_{3}}{p}} \\
\varepsilon \sqrt{1-\frac{m}{E_{\varepsilon}-\alpha m}} \sqrt{1-s \frac{p_{3}}{p}} e^{i \delta}
\end{array}\right),
$$

where neutrino energy is

$$
E_{\varepsilon}=\varepsilon \sqrt{\mathbf{p}^{2}\left(1-s \alpha \frac{m}{p}\right)^{2}+m^{2}}+\alpha m
$$

(here $p$ and $m$ are the neutrino momentum and mass, and $\delta=\arctan p_{2} / p_{1}$ ). The matter density parameter $\alpha$ depends on the type of neutrino and matter composition. For neutrinos of different flavours and matter composed of electrons, neutrons and protons we get

$$
\alpha_{\nu_{e}, \nu_{\mu}, \nu_{\tau}}=\frac{1}{2 \sqrt{2}} \frac{G_{F}}{m}\left(n_{e}\left(4 \sin ^{2} \theta_{W}+\varrho\right)+n_{p}\left(1-4 \sin ^{2} \theta_{W}\right)-n_{n}\right),
$$

where $\varrho=1$ for the electron neutrino $\nu_{e}$, and $\varrho=-1$ in the case of the muon $\nu_{\mu}$ or tau $\nu_{\tau}$ neutrinos. The value $\varepsilon= \pm 1$ splits the solutions into two classes that in the limit of vanishing matter density, $\alpha \rightarrow 0$, represent the positive- and negative-frequency solutions of the Dirac equation in vacuum.

Let us discuss in some detail the obtained neutrino energy spectrum in matter (6). The neutrino dispersion relations in matter exhibits a very fascinating feature (see also [9, 10]): the neutrino energy may has a minimum at non-zero momentum. It may also happen that the neutrino group and phase velocities are oppositely directed. For the fixed value of the neutrino momentum $\mathbf{p}$, there are four different energy values which are characterized by four different combinations of the helicity $s= \pm 1$ and energy sign $\varepsilon= \pm 1$ :

$E^{s=+1}=\sqrt{\mathbf{p}^{2}\left(1-\alpha \frac{m}{p}\right)^{2}+m^{2}}+\alpha m, \quad E^{s=-1}=\sqrt{\mathbf{p}^{2}\left(1+\alpha \frac{m}{p}\right)^{2}+m^{2}}+\alpha m$,

$\tilde{E}^{s=+1}=\sqrt{\mathbf{p}^{2}\left(1-\alpha \frac{m}{p}\right)^{2}+m^{2}}-\alpha m, \quad \tilde{E}^{s=-1}=\sqrt{\mathbf{p}^{2}\left(1+\alpha \frac{m}{p}\right)^{2}+m^{2}}-\alpha m$.

The first pair of energies is for the positive and negative-helicity neutrino states, and the second pair is for the corresponding antineutrino states. From the obtained energy spectrum we conclude [23] that several interesting phenomena may appear when neutrino moves in the vicinity of the interface between media with different densities and, in particular, between vacuum and medium with finite density. As it follows from (8) and (9), the forbidden energy zone for neutrino and antineutrino states in vacuum $-m<E<m$ is shifted to $\alpha m-m<E<\alpha m+m$ in the presence of 
matter. Consequently, a neutrino moving in vacuum with the energy in the range $\alpha m-m<E<\alpha m+m$ can not penetrate into the medium because the corresponding energy range is forbidden in matter, and thus it will be reflected from the interface. The analogous effect of reflection can appear at the interface between media with different densities. Moreover, under certain conditions it may happen that the neutrino (or antineutrino) can not escape from a dense medium into the vacuum, so that the neutrino trapping effect in a dense object may exist (see also[13]). The similar analysis of the neutrino energy spectra in matter shows that there may also exist the processes of the neutrino-antineutrino pair annihilation [9, 10] as well as the spontaneous neutrino pair creation [14] in media.

Note that the obtained energy spectrum of the flavour neutrinos with different helicities in the presence of matter provides the correct description of the neutrino flavour and spin oscillations resonance amplification in matter. Indeed, as it follows from (66) and (77), the energies of the relativistic active electron and muon neutrinos are

$$
E_{\nu_{e}, \nu_{\mu}}^{s=-1} \approx E_{0}+2 \alpha_{\nu_{e}, \nu_{\mu}} m_{\nu_{e}, \nu_{\mu}},
$$

where $m_{\nu_{e}}$ and $m_{\nu_{\mu}}$ are the masses of the electron and muon neutrinos. The energy difference for the two flavour neutrinos is

$$
\Delta E=E_{\nu_{e}}^{s=-1}-E_{\nu_{\mu}}^{s=-1}=\sqrt{2} G_{F} n_{e},
$$

that provides the standard expression for the MSW effect [1, 2]. If one considers the neutrino spin-flavour oscillations $\nu_{e_{L}} \leftrightarrow \nu_{\mu_{R}}$ then the correspondent difference is

$$
\Delta E=E_{\nu_{e}}^{s=-1}-E_{\nu_{\mu}}^{s=+1}=\sqrt{2} G_{F}\left(n_{e}-\frac{1}{2} n_{n}\right),
$$

in agreement with [3, 4].

\section{Quantum theory of neutrino spin light in matter}

The $S L \nu$ in matter originates from the quantum electromagnetic transition between the two neutrino "energy levels" in matter. Therefore, the most consistent theory of this phenomena can be obtained within the quantum treatment [23]-[26] based on the use of the modified Dirac equation (44) exact solutions. Note that the radiation, which is similar to $S L \nu$ in matter, can be also emitted by a neutrino moving in the presence of a magnetic field [29].

The transition amplitude between the neutrino initial $\psi_{i}$ and final $\psi_{f}$ states can be written in the following form:

$$
S_{f i}=-\mu \sqrt{4 \pi} \int d^{4} x \bar{\psi}_{f}(x)\left(\hat{\boldsymbol{\Gamma}} \mathbf{e}^{*}\right) \frac{e^{i k x}}{\sqrt{2 \omega L^{3}}} \psi_{i}(x), \quad \hat{\boldsymbol{\Gamma}}=i \omega\left\{[\boldsymbol{\Sigma} \times \boldsymbol{\kappa}]+i \gamma^{5} \boldsymbol{\Sigma}\right\},
$$

where $\mu$ is the neutrino magnetic moment, $k^{\mu}=(\omega, \mathbf{k})$ and $\mathbf{e}^{*}$ are the momentum and polarization of the emitted photon, and $\kappa=\mathbf{k} / \omega$ is the unit vector in the direction of the photon radiation. Let us consider the case of matter composed of electrons (for 
simplicity we neglect possible effects of matter motion and polarization). Then from the law of energy-momentum conservation,

$$
E=E^{\prime}+\omega, \quad \mathbf{p}=\mathbf{p}^{\prime}+\mathbf{k},
$$

it follows that the $S L \nu$ radiation arises in the transition of the initial neutrino state with negative helicity $s_{i}=-1$ to the final state with positive helicity $s_{f}=+1$. For the emitted photon energy we get

$$
\omega=\frac{2 \alpha m p[(E-\alpha m)-(p+\alpha m) \cos \theta]}{(E-\alpha m-p \cos \theta)^{2}-(\alpha m)^{2}},
$$

where the angle $\theta$ gives the direction of the radiation in respect to the initial neutrino momentum p. For the $S L \nu$ radiation rate and total power we get, respectively,

$$
\Gamma=\mu^{2} \int_{0}^{\pi} \frac{\omega^{3}}{1+\tilde{\beta}^{\prime} y} S \sin \theta d \theta, \quad I=\mu^{2} \int_{0}^{\pi} \frac{\omega^{4}}{1+\tilde{\beta}^{\prime} y} S \sin \theta d \theta,
$$

where

$$
\begin{aligned}
& S=\left(\tilde{\beta} \tilde{\beta}^{\prime}+1\right)(1-y \cos \theta)-\left(\tilde{\beta}+\tilde{\beta}^{\prime}\right)(\cos \theta-y), \\
& \tilde{\beta}=\frac{p+\alpha m}{E-\alpha m}, \quad \tilde{\beta}^{\prime}=\frac{p^{\prime}-\alpha m}{E^{\prime}-\alpha m}, \quad E^{\prime}=E-\omega, \quad p^{\prime}=K \omega-p, \\
& y=\frac{\omega-p \cos \theta}{p^{\prime}}, \quad K=\frac{E-\alpha m-p \cos \theta}{\alpha m} .
\end{aligned}
$$

In the relativistic neutrino momentum case, $p \gg m$, and for different values of the matter density parameter $\alpha$ from (16) and we have the following limiting values [23]-[26]:

$\Gamma=\left\{\begin{array}{cl}\frac{64}{3} \mu^{2} \alpha^{3} p^{2} m, \\ 4 \mu^{2} \alpha^{2} m^{2} p, \\ 4 \mu^{2} \alpha^{3} m^{3},\end{array} \quad I=\left\{\begin{array}{cl}\frac{128}{3} \mu^{2} \alpha^{4} p^{4}, & \text { for } \alpha \ll \frac{m}{p}, \\ \frac{4}{3} \mu^{2} \alpha^{2} m^{2} p^{2}, & \text { for } \frac{m}{p} \ll \alpha \ll \frac{p}{m}, \\ 4 \mu^{2} \alpha^{4} m^{4}, & \text { for } \alpha \gg \frac{p}{m} .\end{array}\right.\right.$

In the opposite case of "non-relativistic" neutrinos, $p \ll m$, we get:

$\Gamma=\left\{\begin{array}{cl}\frac{64}{3} \mu^{2} \alpha^{3} p^{3}, \\ \frac{512}{5} \mu^{2} \alpha^{6} p^{3}, \\ 4 \mu^{2} \alpha^{3} m^{3},\end{array} \quad I=\left\{\begin{array}{cl}\frac{128}{3} \mu^{2} \alpha^{4} p^{4}, & \text { for } \alpha \ll 1, \\ \frac{1024}{3} \mu^{2} \alpha^{8} p^{4}, & \text { for } 1 \ll \alpha \ll \frac{m}{p}, \\ 4 \mu^{2} \alpha^{4} m^{4}, & \text { for } \alpha \gg \frac{m}{p} .\end{array}\right.\right.$

It can be seen that in the case of a very dense matter the values of the rate and total power are mainly determined by the density. Note that the obtained above results in the case of small densities are in agreement with the studies of the neutrino spin light performed on the basis of the quasi-classical approach [18]. The $S L \nu$ characteristics in the case of matter with "moderate" densities (the second lines of (20) ) were also obtained in [30].

One can estimate the average emitted photon energy $\langle\omega\rangle=I / \Gamma$ with the use of the obtained above values of the rate and total power (20) and (21) for different matter densities. In the two case $(p \gg m$ and $p \ll m)$, we get, respectively,

$\langle\omega\rangle \simeq\left\{\begin{array}{cc}2 \alpha \frac{p^{2}}{m}, & \text { for } \alpha \ll \frac{m}{p}, \\ \frac{1}{3} p, & \text { for } \frac{m}{p} \ll \alpha \ll \frac{p}{m}, \\ \alpha m, & \text { for } \alpha \gg \frac{p}{m},\end{array} \quad\langle\omega\rangle \simeq\left\{\begin{array}{cc}2 \alpha p, & \text { for } \alpha \ll 1, \\ \frac{10}{3} \alpha^{2} p, & \text { for } 1 \ll \alpha \ll \frac{m}{p}, \\ \alpha m, & \text { for } \alpha \gg \frac{m}{p} .\end{array}\right.\right.$ 
To summaries the main properties of the spin light of neutrino in matter, we should like to point out that this phenomenon arises due to neutrino energy dependence in matter on the neutrino helicity state. In media characterized by the positive values of the parameter $\alpha$, the negative-helicity neutrinos (the left-chiral relativistic neutrinos) are converted into the positive-helicity neutrinos (the right-chiral relativistic neutrinos) in the process under consideration. Thus, the neutrino self-polarization effect can appear (see also [19]). From the above estimations for the emitted photon energies it follows that for the relativistic neutrinos moving in very dense matter the $S L \nu$ can be regarded as an effective mechanism for production of the gamma-rays. In this concern we propose (see also [26]) that this mechanism can be relevant to the fireball model of GRBs and to the description of the gamma-rays from the collapses or coalescence processes of neutron stars, as well as for the radiation originated during a neutron star being "eaten up" by the black hole at the center of our Galaxy.

\section{Conclusion}

Motivated by the need of elaboration of the quantum theory of the spin light of neutrino in matter, we have studied in detail the exact solutions of the Dirac equation for neutrinos moving in the background matter. In application of the obtained exact solutions to the $S L \nu$ process we have clearly demonstrated that it is possible to develop a rather powerful method of investigation of different neutrino processes in matter, which is similar to the Furry representation of quantum electrodynamics in external electromagnetic fields. Note that in addition to the obtained neutrino wave functions in matter it is also important to get the explicit expression for the corresponding neutrino Green function [28].

To conclude, we should like to argue that the developed quantum approach to description of neutrinos in the presence of background matter can be also applied to electrons (and other particles) moving in matter. Let us consider an electron propagating in electrically neutral matter composed of neutrons, electrons and protons. This situation can be realized, for instance, when electrons move in matter of a neutron star. We suppose that there is a macroscopic amount of the background particles in the scale of an electron de Broglie wave length. Then the addition to the electron effective interaction Lagrangian is

$$
\Delta L_{e f f}^{(e)}=-f^{\mu}\left(\bar{e} \gamma_{\mu} \frac{1-4 \sin ^{2} \theta_{W}+\gamma^{5}}{2} e\right)
$$

where $f^{\mu}$ is determined by (11) and (2) with $\delta_{e f}=0$. The modified Dirac equation for the electron wave function in matter is

$$
\left\{i \gamma_{\mu} \partial^{\mu}-\frac{1}{2} \gamma_{\mu}\left(1-4 \sin ^{2} \theta_{W}+\gamma_{5}\right) \tilde{f}^{\mu}-m_{e}\right\} \Psi_{e}(x)=0
$$

where

$$
\tilde{f}^{\mu}=-f^{\mu}=\frac{G_{F}}{\sqrt{2}}\left(j_{n}^{\mu}-\lambda_{n}^{\mu}\right) .
$$


The corresponding electron energy spectrum in the case of unpolarized matter at rest is given by

$$
E_{\varepsilon}^{(e)}=\varepsilon \sqrt{\mathbf{p}_{e}^{2}\left(1-s_{e} \alpha_{n} \frac{m_{e}}{p_{e}}\right)^{2}+m_{e}^{2}}+c \alpha_{n} m_{e}, \quad \alpha_{n}=\frac{1}{2 \sqrt{2}} G_{F} \frac{n_{n}}{m_{e}}
$$

where $c=1-4 \sin ^{2} \theta_{W}$ and the notations for the electron mass, momentum, helicity and sign of energy are similar to those used in Section 2 for the case of neutrino. The exact solutions of this equation open a new method for investigation of different quantum processes which can appear when electrons propagate in matter. On this basis, we predict a mechanism of the electromagnetic radiation by an electron moving in matter which we term the "spin light of electron in matter" (SLe). Note that the similar term ("spin light of electron in magnetic field") was used previously for the magnetic moment dependent contribution to the radiation of an electron in a magnetic field [32]. The SLe photon energy, obtained from the energy conservation law, is given by

$$
\omega_{S L e}=\frac{2 \alpha_{n} m_{e} p_{e}\left[\left(E^{(e)}-c \alpha_{n} m_{e}\right)-\left(p_{e}+\alpha_{n} m_{e}\right) \cos \theta_{S L e}\right]}{\left(E^{(e)}-c \alpha_{n} m_{e}-p_{e} \cos \theta_{S L e}\right)^{2}-\left(\alpha_{n} m_{e}\right)^{2}},
$$

where the angle $\theta_{S L e}$ gives the direction of the radiation in respect to the initial electron momentum $\mathbf{p}_{e}$. In the case of relativistic electrons and small values of the matter density parameter $\alpha_{n}$ the photon energy is

$$
\omega_{S L e}=\frac{\beta_{e}}{1-\beta_{e} \cos \theta_{S L e}} \omega_{0}, \quad \omega_{0}=\frac{G_{F}}{\sqrt{2}} n_{n} \beta_{e},
$$

here $\beta_{e}$ is the electron speed in vacuum. From this expressions we conclude that for the relativistic electrons the energy range of the $S L e$ may even extend up to energies peculiar to the spectrum of gamma-rays. We also predict the existence of the electronspin polarization effect in this process. Finally, from the order-of-magnitude estimation, we expect that the ratio of rates of the $S L \mathrm{e}$ and the $S L \nu$ in matter is

$$
R=\frac{\Gamma_{S L e}}{\Gamma_{S L \nu}} \sim \frac{e^{2}}{\omega^{2} \mu^{2}}
$$

that gives $R \sim 10^{18}$ for the radiation in the range of gamma-rays, $\omega \sim 5 \mathrm{MeV}$, and for the neutrino magnetic moment $\mu \sim 10^{-10} \mu_{0}$. Thus, we expect that in certain cases the $S L e$ in matter would be more effective than the $S L \nu$. For the detailed study of the $S L e$ in matter see [31].

I would like to thank Emilio Elizalde and Sergei Odintsov for the invitation to participate to the Seventh Workshop on Quantum Field Theory under the Influence of External Conditions. I am also thankful to all of the organizers of this workshop for their hospitality.

\section{References}

[1] Wolfenstein L 1978 Phys.Rev. D 172369

[2] Mikheyev S and Smirnov A 1985 Sov.J.Nucl.Phys. 42913

[3] Akhmedov E 1988 Phys.Lett. B 21364 
[4] Lim C-S and Marciano W 1988 Phys.Rev. D 371368

[5] Raffelt G G 1995 Stars as Laboratories for Fundamental Physics (Chicago: The University of Chicago Press) p. 304

[6] Mannheim P 1988 Phys.Rev. D 371935

[7] Nötzold D and Raffelt G 1988 Nucl.Phys. B 307924

[8] Nieves J 1989 Phys.Rev. D 40866

[9] Chang L N and Zia R K 1988 Phys.Rev. D 381669

[10] Pantaleone J 1991 Phys.Lett. B 268227

Pantaleone J 1992 Phys.Rev. D 46510

Kiers K and Weiss N 1997 Phys.Rev. D 565776

Kiers K and Tytgat M 1998 Phys.Rev. D 575970

[11] Oraevsky V, Semikoz V and Smorodinsky Ya 1989 Phys.Lett. B 227255

[12] Haxton W and Zhang W-M 1991 Phys.Rev. D 432484

[13] Loeb A 1990 Phys.Rev.Lett. 64115

[14] Kachelriess M 1998 Phys.Lett. B 42689

Kusenko A and Postma M 2002 Phys.Lett. B 545238

[15] Koers H B J 2004 Preprint hep-ph/0409259

[16] Berezhiani Z and Vysotsky M 1987 Phys.Lett. B 199281

Berezhiani Z and Smirnov A 1989 Phys.Lett. B 220279

Giunti C, Kim C W, Lee U W and Lam W P 1992 Phys.Rev. D 451557

[17] Berezhiani Z and Rossi A 1994 Phys.Lett. B 336439

[18] Egorov A, Lobanov A and Studenikin A 2000 Phys.Lett. B 491137

Lobanov A and Studenikin A 2001 Phys.Lett. B 51594

Dvornikov M and Studenikin A 2001 Phys.Atom.Nucl. 641624

Dvornikov M and Studenikin A 2002 JHEP 09016

Studenikin A 2004 Phys.Atom.Nucl. 67993

Dvornikov M and Studenikin A 2004 Phys.Atom.Nucl. 67719

Studenikin A 2005 Nucl.Phys.(Proc.Suppl) B 143570

[19] Lobanov A and Studenikin A 2003 Phys.Lett. B 56427

Lobanov A and Studenikin A 2004 Phys.Lett. B 601171

[20] Fujikawa K and Shrock R 1980 Phys.Rev.Lett. 45963

[21] Dvornikov M and Studenikin A 2004 Phys.Rev. D 69073001

Dvornikov M and Studenikin A 2004 JETP 99254

[22] Dvornikov M, Grigoriev A and Studenikin A 2005 Int.J.Mod.Phys. D 14309

[23] Studenikin A and Ternov A 2005 Phys.Lett. B 608107 Preprint hep-ph/0410297 Preprint hep-ph/0412408

[24] Studenikin A and Ternov A 2004 Proc. of the 13th Int. Seminar on High Energy Physics "Quarks2004" (Moscow)(Publ. Department of Institute of Nuclear Physics RAS, Eds.: Levkov D, Matveev V and Rubakov V) Preprint hep-ph/0410296

Grigoriev A, Studenikin A and Ternov A 2005 Particle Physics in Laboratory, Space and Universe (Singapore) (World Scientific, Ed.: Studenikin A) ) 55 Preprint hep-ph/0502210

[25] Grigoriev A, Studenikin A and Ternov A 2005 Grav. 83 Cosm. 11132 (Preprint hep-ph/0502231)

[26] Grigoriev A, Studenikin A and Ternov A 2005 Phys.Lett. B 622199

[27] Grigoriev A, Studenikin A and Ternov A 2006 Phys.Atom.Nucl. 69 (in press)

[28] Pivovarov I and Studenikin A 2006 PoS(HEP2005) 191 Preprint hep-ph/0512031

[29] Borisov A V, Zhukovskii V Ch and Ternov A I 1988 Sov.Phys.J. 31228

[30] Lobanov A 2005 Phys.Lett. B 619136

[31] Shinkevich S, Studenikin A, Ternov A and Trofimov I 2006 to be published

[32] Bordovitsyn V A, Ternov I M, Bagrov V G 1995 Physics - Uspekhi 381037 